\title{
Influences of Different Inoculation Methods on Fluorescence Characteristics of DOM in Solid Waste Composting
}

\author{
Bo Yao ${ }^{1, a}$, Chunming $\mathrm{Hu}^{2, \mathrm{~b}}$, Qingquan $\mathrm{Liu}^{1, \mathrm{c}}$, Beidou $\mathrm{Xi}^{2, \mathrm{~d}}$ \\ ${ }^{1}$ Key Laboratory for Mechanics in Fluid Solid Coupling System, Institute of Mechanics, \\ Chinese Academy of Sciences, Beijing 100190, China \\ ${ }^{2}$ Chinese Research Academy of Sciences, Beijing 100012, China \\ ayaobo@imech.ac.cn, bhuchunming666@163.com, 'qqliu@imech.ac.cn, 'xibeidou@263.net
} Keywords: solid waste composting; inoculation methods; dissolved organic matter; synchronous
fluorescence spectra.

\begin{abstract}
In order to determine better inoculation methods of compound microorganisms in solid waste composting, biochemical parameters and dissolved organic matter (DOM) during the process of composting with five different inoculation methods were tested and analyzed. The results show that temperature, $\mathrm{pH}$ value, and synchronous fluorescence spectra of DOM in the composting piles of the five deals exhibits a certain trend with composting time increasing, in which macromolecules with complex structures and humic substances gradually increase. Overall, the temperature increases first and then decreases, and $\mathrm{pH}$ value is nearly stable at slightly alkaline range by the end of composting. Complex macromolecules and humic substances increase, and DOM properties tend to stabilize. Meanwhile, humification index (IA/IC, \%PLF, and \%HLF) of the five deals of compost also experienced significant changes. With the decrease of protein-like substances, the content of humus correspondingly increase, i.e. with composting time increasing, low-molecular-weight organic matter in the compost gradually transforms into stable high-molecular-weight humic substances, which suggests increasing aromaticity and distinctive complexity of DOM. From a comprehensive comparison of the relevant parameters and indicators, the deal of inoculating compound microorganisms at the start time or after the high-temperature phase could notably speed up the humification process in solid waste composting.
\end{abstract}

\section{Introduction}

Microbial inoculation can significantly promote the reaction progress in composting of domestic solid waste and shorten the composting cycle [1]. Therefore, researches on inoculation of high-efficient agents to accelerate the composting process and to improve the quality of compost have important practical significances [2]. However, some key problems may still exist in the application of microbial inoculation techniques in composting, such as the suitable time for inoculation. It is of vital importance to select appropriate time to inoculate the agents, as it determines whether the inoculated bacterial can adapt to the composting environment, and to what extent they could improve the efficiency of composting [3]. Although microbial inoculation techniques in solid waste composting have been widely used in recent years, influences of inoculation time on the spectral characteristics of DOM in the composting process and the maturity degree of compost are still not very clear [4].

Compared to direct detections of materials in solid phase during the composting process, observations on changes of active functional groups in dissolved organic matters (DOM) can better reflect the compost maturity degree [5]. It is important to understand the structural properties of DOM, as it can not only reflect transformation of materials in the composting process, but also can be significant indicators on maturity degree and quality of the compost.

At present, the use of spectral scanning means to carry out analysis on molecular structures in the composting process and to determine maturity and quality of the compost is one of the main aspects in composting studies. As there are fluorescent substances such as phenolic structures and aromatic 
carboxylic acids in the DOM, fluorescent spectrum techniques can be an effective mean in such field [6]. Comparing to conventional emission and excitation spectra, synchronous fluorescence spectroscopy can give more information about the structures and functional groups of DOM, and is considered as the molecular fingerprinting [7].

In this paper, composting experiments with different inoculation methods were carried out, to investigate how inoculation time affects biochemical characteristics and transformations of organic matters in composting. Parameters such as pile temperature and $\mathrm{pH}$ value were analyzed, and fluorescent spectrums of DOM during the composting process were also investigated, helping find better inoculation methods which could improve the quality of municipal solid waste compost and maturity.

\section{Materials and Methods}

\section{Composting materials}

The materials for composting are composed of domestic garbage, sawdust, and dried turf grass clippings. The properties of the materials are shown in table 1 . The ratio (by weight) of the three materials is 360: 13: 3, with initial C: $\mathrm{N}$ ratio of 28.33 and moisture of $63.86 \%$.

Table 1. Basic properties of the composting materials

\begin{tabular}{cccccc}
\hline Materials & $\begin{array}{c}\text { Total } \\
\text { Carbon[mg/g] }\end{array}$ & $\begin{array}{c}\text { Total } \\
\text { Nitrogen[mg/g] }\end{array}$ & $\mathrm{C}: \mathrm{N}$ & $\mathrm{pH}$ & Moisture[\%] \\
\hline $\begin{array}{c}\text { domestic } \\
\text { garbage }\end{array}$ & 30.24 & 2.70 & 11.20 & 4.40 & $66.7 \%$ \\
$\begin{array}{c}\text { sawdust } \\
\text { grass } \\
\text { clipping }\end{array}$ & 60.82 & 0.12 & 506.8 & 8.07 & $<5.0 \%$ \\
\hline
\end{tabular}

\section{Composting methods}

A two-room- composting equipment [4] was used in our experiments. And five inoculation methods for composite microbial agent were set according to composting temperature and phase, which were inoculation at the begening of composting (IcB), inoculation at the high-temperature phase (IcH), inoculation at the phase after the high-temperature phase (IcA), inoculation at the maturity phase (IcM), and without incoulation (IcT). Here, the high temperature phase is denfined as the period when the temperautre of the compositing pile is higher than $60^{\circ} \mathrm{C}$. The concentration of agent for inocultion is $1 \times 10^{8} \mathrm{CFU} / \mathrm{mL}$, and the inoculum size is $0.5 \mathrm{~mL}$ agent per $\mathrm{kg}$ of wet-weight composting materials. Sampling and analyzing methods

Pile temperature during the composting process was recorded every day. Compositing samples were collected every other day, and about $500 \mathrm{~g}$ solid was collected from the composting piles from different inoculation treatments each time. All samples collected were then stored in refrigerator below $-20{ }^{\circ} \mathrm{C}$ before further analysis.

\section{DOM extraction and fluorescent spectrum scanning}

Two hundred grams of fresh composting sample was added to $2 \mathrm{~L}$ of distilled water. The suspension was shaken at $200 \mathrm{r} / \mathrm{min}$ for $16 \mathrm{~h}$ and centrifuged at $10,000 \mathrm{~g}$ for $30 \mathrm{~min}$. The supernatant was then filtered through a $0.45 \mathrm{pm}$ membrane filter, and the filtrate (i.e. DOM) was diluted into a uniformed DOC concentration of $10 \mathrm{mg} / \mathrm{L}$ for spectral analysis.

Synchronous-scan spectra were measured by scanning simultaneously over the range from $300 \mathrm{~nm}$ to $450 \mathrm{~nm}$, with optimized wavelength difference: $\Delta \lambda=\lambda_{e m}-\lambda_{e x}=18 \mathrm{~nm}$. 


\section{Results and Discussions}

\section{Pile temperature}

Pile temperature is a visual representation for the composting reaction process, and its variations can significantly affect microbial activities, thus it is an important indicator for composting process. Fig. 1 gives the variations of pile temperature throughout the composting process. Comparing the five treatments, it could be seen that pile temperature rises relatively slow for the treatment of IcK and IcM, but fast for IcB and IcA. The treatment IcB also has a relatively long high-temperature phase of about 13 days, indicating more intense microbial activity in the composting pile. The treatment of IcH also has a relatively fast increase of temperature and long high-temperature phase, but its pile temperature decreases faster than the others, with only one temperature peak.

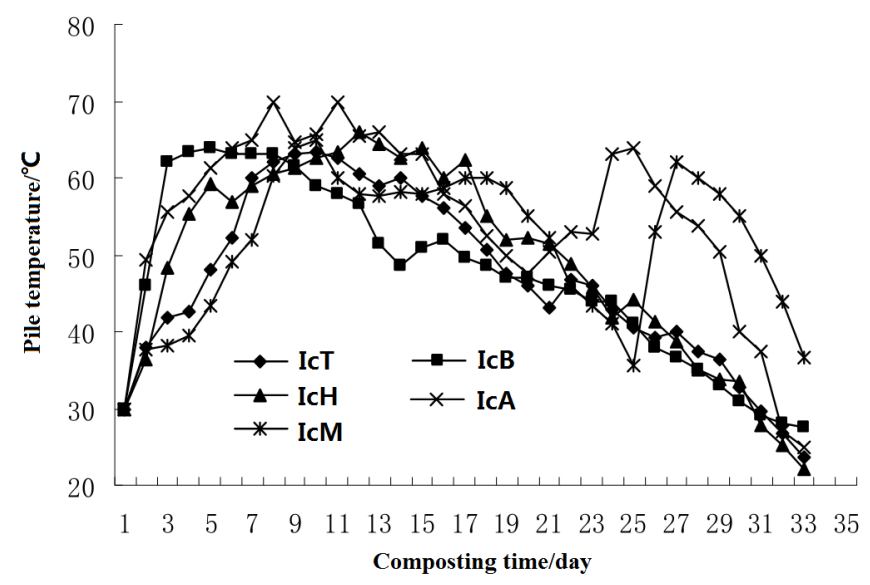

Fig.1 Changes of temperature through the composting process

It could also be seen from Fig. 1 that there is a secod temperature peak after the high-temperature phase for the treamtment of IcA and IcM. This is mainly due to that when the pile temperature droped, the activity of indigenous bacteria decreased, and a number of harmful microorganisms died in the high-temperature phase. As a result, the environment is very suitbale for the inoculated composite bacterials, which favores their fast growth and causes the second phase for decomposition of biodegradable organic matters and resulted in the second temperature peak. However, as most of the degradable organic matters have been depleted in the first phase, the second high-temperature phase could only keep a few days and then the temperature droped very fast.

\section{pH value}

The variations of $\mathrm{pH}$ value for the composting materials are shown in Fig.2. It could be seen that $\mathrm{pH}$ value shows an increasing trend during the composting process for all the five treatments, and a three-stage variation could be found. In the early days (within about the first 9 days), the $\mathrm{pH}$ value changes very slightly, rising from the initial value of 4.52 to about 5.00. It is mainly due to that during this period, small molecular weight organic acids decomposed from the composting materials were utilized by microorganisms, and a part of the generated $\mathrm{CO}_{2}$ and $\mathrm{CH}_{4}$ volatilized to the air. Besides, the $\mathrm{NH}_{+}-\mathrm{N}$ from microbial decomposition did not completely used by microorganisms. In the second phase (from about the $10^{\text {th }}$ day to the $30^{\text {th }}$ day), the $\mathrm{pH}$ value increases very fast, rising up to about 7.00. In this period, microbial activity is very strong, and organic matters in the composting materials are fast degraded. Organic acids are consumed and alkaline substances such as $\mathrm{NH} 4+$ accumulated, leading to increase of the $\mathrm{pH}$ value. And in the last period (after about 30 days), the $\mathrm{pH}$ value come to be stable as a result of depletion of organic matters in the composting materials. And the final $\mathrm{pH}$ values for the five treatments are between 7.33 and 8.12, in which the treatment IcA has the largest value of 8.12 . 


\section{Synchronous fluorescence spectra}

Synchronous fluorescence spectra can give more information on structures and functional groups in the DOM than excitation and emission spectrum do. It represents the combinations of several different fluorescent groups in the DOM, such as protein-like substances and humic acids [8]. Fig.3 shows the synchronous fluorescence spectra for the five treatments and the one before composting. It could be seen that the shapes of the five spectrums are nearly the same, but their peak intensities are obviously different. In the spectrum before composting, there is only a strong peak near $277 \mathrm{~nm}$. But two another peaks appear in the spectrums of the five treatments after composting, one is near $341 \mathrm{~nm}$, and the other near $327 \mathrm{~nm}$. It is suggested that the peak near $277 \mathrm{~nm}$ represents protein substances, which is mainly composed of materials with low aromatization, simple structure, and small molecular condensation [9]. These substances are mainly from degradation of aromatic amino acids by recent microbial activities. And the fluorescent peaks near $341 \mathrm{~nm}$ and $373 \mathrm{~nm}$ in the spectrum are mainly from dissolved humic substances especially fulvic-like substances.

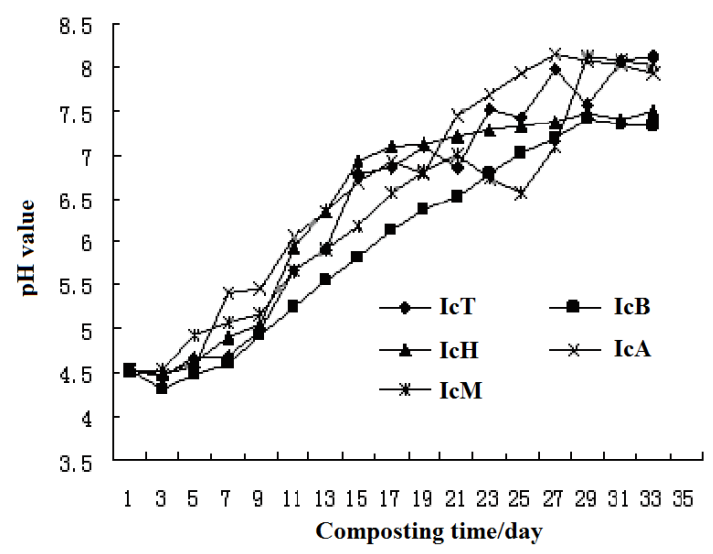

Fig.2 Time variations of $\mathrm{pH}$ values for the five composting treatments

It could also be seen from Fig.3 that comparing to the spectrum before composting, wavelength for the peak near $277 \mathrm{~nm}$ shifts to the right (i.e. red-shift) in the spectrum after composting, in which the wavelengths for IcB, IcH, IcA, IcM, and IcT are $285 \mathrm{~nm}, 281 \mathrm{~nm}, 284 \mathrm{~nm}, 282 \mathrm{~nm}$, and $279 \mathrm{~nm}$, respectively. This red-shift occurred in fluorescent spectra is mainly due to concentrating and aggregating process of fluorescent organic molecules, and suggests that after composting, the content of fulvic-like acids increases in the DOM, as well as that the degree of aromatization become higher. And the degree of red-shift to some extent indicates the maturity of the compost. Comparing the five treatments, inoculation at the beginning (IcB) and after the high-temperature phase (IcA) has larger red-shift than the other treatments, which could more significantly improve humification process of the composting materials.

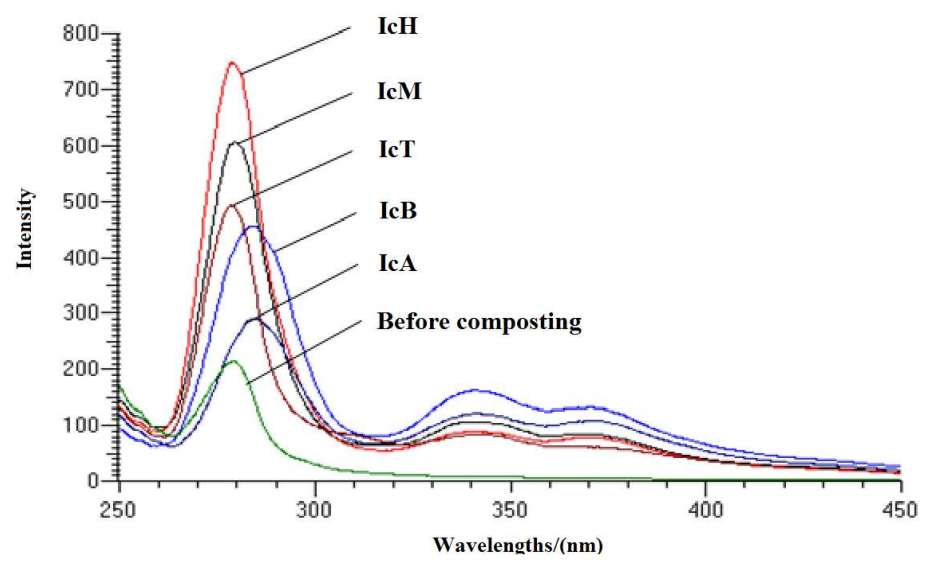

Fig.3 Synchronous fluorescence spectra for DOM in composting samples 
As the red-shift in the spectrum could be an indicator of humification and maturity, some researchers proposed to use humification index to characterize humification degree of DOM in composting [10]. In this paper, humification degree of the compost is represented by $\mathrm{r}(\mathrm{A}, \mathrm{C})=\mathrm{IA} / \mathrm{IC}$, in which IA and IC are peak intensities near $370 \mathrm{~nm}$ and $340 \mathrm{~nm}$ in the synchronous scan spectra, respectively. Relative content of protein-like substances and humic acids are separately represented by $\% \mathrm{PLF}$ and $\% \mathrm{HLF}$, which could be calculated as follows:

$$
\begin{aligned}
& \% P L F=A_{250-300} /\left(A_{250-300}+A_{300-550}\right) \\
& \% H L F=A_{300-550} /\left(A_{250-300}+A_{300-550}\right)=1-\% P L F
\end{aligned}
$$

where $A_{250-300}$ is the area from $250 \mathrm{~nm}$ to $300 \mathrm{~nm}$ in the synchronous scan spectra, and $A_{300-550}$ is the area from $300 \mathrm{~nm}$ to $550 \mathrm{~nm}$.

The calculated $\mathrm{r}(\mathrm{A}, \mathrm{C})$ and \%PLE values are shown in Table 2 and Table 3. Generally, $\mathrm{r}(\mathrm{A}, \mathrm{C})$ values for the five treatments increase with composting time. In the meantime, values of \%PLF decrease, and \%HLF increase $(\% \mathrm{HLF}=1-\% \mathrm{PLF})$, indicating that protein-like substances in the composting materials decrease along composting time. By the end of composting, $r(\mathrm{~A}, \mathrm{C})$ values for the five treatments IcT, IcB, IcH, IcA, and IcM are 0.744, 0.92, 0.82, 0.89, and 0.81, respectively. And their related values of \%HLF are 12.17, 21.32, 14.77, 22.50, and 15.59, respectively. This suggests that as composting going, the content of humus increases, in which low molecular-weight organic matters in the composting materials were transformed into stale and high molecular-weight humic substances by microbial activities.

Table 2. Variations of $\mathrm{r}(\mathrm{A}, \mathrm{C})$ values for the five treatments

\begin{tabular}{cccccc}
\hline Time & IcT & IcB & IcH & IcA & IcM \\
\hline $7 \mathrm{~d}$ & 0.66 & 0.72 & 0.7 & 0.7 & 0.68 \\
$15 \mathrm{~d}$ & 0.7 & 0.78 & 0.77 & 0.72 & 0.7 \\
$24 \mathrm{~d}$ & 0.73 & 0.86 & 0.79 & 0.82 & 0.73 \\
$33 \mathrm{~d}$ & 0.744 & 0.92 & 0.82 & 0.89 & 0.81 \\
\hline
\end{tabular}

Table 3. Variations of \%PLF values for the five treatments

\begin{tabular}{cccccc}
\hline Time & IcT & IcB & IcH & IcA & IcM \\
\hline 0d & 97.47 & 97.47 & 97.47 & 97.47 & 97.47 \\
7d & 96.13 & 93.2 & 94.91 & 95.05 & 95.95 \\
15d & 93.94 & 89.83 & 88.14 & 87.34 & 93.35 \\
24d & 89.79 & 82.43 & 86.72 & 80.94 & 89.31 \\
33d & 87.83 & 78.68 & 85.23 & 77.5 & 86.41 \\
\hline
\end{tabular}

It could also be seen that inoculation in composting could significantly improve the humification process, as vale of \%HLF for the treatment without inoculation (IcT) is obviously smaller than those of the other four treatments with inoculation. Comparing the five treatments, values of $\mathrm{r}(\mathrm{A}, \mathrm{C})$ and \%HLF for IcA and IcB increased faster than the others'. This may be due to that when inoculation at the beginning, the inoculating microorganisms could be able to play a role in bio-degradation of organic materials from the beginning of composting, and could last a long period of microbial activity. While for the treatment of inoculation after the high-temperature phase (IcA), rich nutrients left from previous bio-degradation process and suitable pile temperature could enable the inoculated agents to adapt the new environment quickly, leading to a second bio-degradation process. This could result in another peak of pile temperature (see Fig. 1), and promote the humification of composting materials. Therefore, comparing the five treatments, IcB and IcA are better than the other treatments in humification of composting materials and maturity of the final composting products. 


\section{Results}

Inoculation of bacterial agents can promote progress of domestic waste composting, and when to inoculate affects humification and maturity of the final compost product. This paper carries out five treatments with different inoculation methods to find out better inoculation time, through analysis on pile temperature, $\mathrm{pH}$ and synchronous scanning spectra of DOM. The main results were:

1) Pile temperature during the composting period increases first and drops fast after the high-temperature phase, and there is a second temperature peak in the treatment IcA and IcM, indicating another bio-degradation peak after inoculation.

2) Variations of $\mathrm{pH}$ value for the five treatments show similar trends, and the final $\mathrm{pH}$ value is between 7.33 and 8.12, in which the treatment IcA has the largest value of 8.12.

3) The values of $\mathrm{r}(\mathrm{C}, \mathrm{A})$ at the end of composting for the five treatments IcT, IcB, IcH, IcA, and IcM are $0.744,0.92,0.82,0.89$, and 0.81 , respectively; and values of \%HLF are 12.17, 21.32, 14.77, 22.50 , and 15.59 , respectively.

4) Comparing the five treatments, inoculation at the beginning (IcB) and after the high-temperature phase (IcA) are better than the other treatments, leading to compost product with higher humification and maturity.

\section{Acknowledgements}

This paper was financially supported by National Natural Science Foundation of China (11202217), National Natural Science Foundation of China (50878201), and Natural Science Foundation of Heilongjiang Province (C200917).

\section{References}

[1] A. H. Vuorinen, M. H. Saharinen. Agr. Ecosyst. Environ., vol. 66 (1997), p.19-29.

[2] R. Barrena, E. Pagans, G. Faltys, A. Sanchez. J. Chem. Technol. Biot., vol. 81(2006), p.420- 425.

[3] D. Huang, G. Zeng, G. Huang, C. Niu. J. Microbiol., vol. 25 (2005), p.60-64.

[4] B. Xi, Z. Wei, Y. Zhao, M. Li, H. Liu. Spectrosc. Spectr. Anal., vol. 28 (2008), p. 2605-2608.

[5] J. Chen, B. Gu, E. J. Leboeuf, H. Pan, S. Dai. Chemosphere, vol. 48(2002), p. 59-68.

[6] N. Senesi. Anal. Chim. Acta, vol. 232(1990), p.77-106.

[7] D. M. McKnight, E. W. Boyer, P. T. Doran. Limnol. Oceanogr, vol. 46(2001), p. 38-48.

[8] J. Hur, D. H. Lee, H. S. Shin. Org. Geochem., vol. 40 (2009), p. 1091-1099.

[9] J. Chen, E. J. Leboeuf, S. Dai, B. Gu. Chemosphere, vol. 50(2003), p. 639-647.

[10] A. P. Wolfe, S. S. Kaushal, J. R. Fuoton. Environ. Sci. Technol., vol. 36 (2002), p. 3217-3223. 